Supplement of Atmos. Chem. Phys., 21, 4471-4485, 2021

https://doi.org/10.5194/acp-21-4471-2021-supplement

(C) Author(s) 2021. CC BY 4.0 License.

(c) (1)

Supplement of

\title{
Temporally resolved sectoral and regional contributions to air pollution in Beijing: informing short-term emission controls
}

Tabish Umar Ansari et al.

Correspondence to: Tabish Umar Ansari (t.u.ansari@rug.nl) and Oliver Wild (o.wild@lancaster.ac.uk)

The copyright of individual parts of the supplement might differ from the article licence. 
Table S1. Summary of the contributions to $\mathrm{PM}_{2.5}$ in Beijing from each one-day pulse in emissions from Beijing, Near-neighbourhood and Far-neighbourhood sources showing the magnitude and timing of the peak contribution and the integrated contribution from each source region

\begin{tabular}{|c|c|c|c|c|c|c|c|c|c|c|c|c|}
\hline \multirow[t]{2}{*}{ Start date } & \multicolumn{3}{|c|}{$\begin{array}{l}\text { Peak contribution } \\
\qquad\left(\mu \mathrm{g} \mathrm{m}^{-3}\right)\end{array}$} & \multicolumn{3}{|c|}{$\begin{array}{l}\text { Time to reach peak } \\
\text { (h) }\end{array}$} & \multicolumn{3}{|c|}{$\begin{array}{l}\text { Length of contribution } \\
\qquad \text { (h) }\end{array}$} & \multicolumn{3}{|c|}{$\begin{array}{l}\text { Integrated contribution } \\
\qquad\left(\mu \mathrm{g} \mathrm{m}^{-3} \mathrm{~h}\right)\end{array}$} \\
\hline & Beijing & Near & Far & Beijing & Near & Far & Beijing & Near & Far & Beijing & Near & Far \\
\hline \multicolumn{13}{|c|}{ Polluted period } \\
\hline $18 \mathrm{Oct}$ & 33.1 & 33.7 & 15.0 & 16 & 30 & 46 & 28 & 44 & 61 & 393 & 386 & 244 \\
\hline 19 Oct & 29.9 & 21.6 & 1.9 & 14 & 24 & 31 & 35 & 36 & 44 & 503 & 320 & 30 \\
\hline 20 Oct & 13.0 & 5.8 & 3.8 & 12 & 21 & 103 & 23 & 116 & 117 & 171 & 108 & 203 \\
\hline 21 Oct & 18.6 & 11.5 & 6.6 & 16 & 33 & 79 & 33 & 101 & 93 & 225 & 560 & 153 \\
\hline 22 Oct & 26.5 & 27.4 & 15.8 & 15 & 39 & 58 & 34 & 93 & 95 & 367 & 913 & 398 \\
\hline 23 Oct & 28.8 & 43.1 & 6.0 & 15 & 36 & 61 & 40 & 71 & 72 & 533 & 795 & 87 \\
\hline 24 Oct & 31.0 & 22.9 & 5.5 & 14 & 37 & 38 & 48 & 48 & 49 & 663 & 226 & 73 \\
\hline $25 \mathrm{Oct}$ & 43.5 & 0.6 & 1.3 & 14 & 103 & 19 & 24 & 66 & 49 & 496 & 1 & 8 \\
\hline 26 Oct & 8.3 & 9.2 & 3.1 & 13 & 34 & 105 & 25 & 45 & 50 & 95 & 69 & 57 \\
\hline 27 Oct & 20.9 & 10.3 & 8.2 & 17 & 33 & 82 & 30 & 91 & 101 & 270 & 462 & 307 \\
\hline \multicolumn{13}{|c|}{ Clean period } \\
\hline $02 \mathrm{Nov}$ & 19.0 & 0.2 & 2.6 & 14 & 33 & 37 & 25 & 30 & 74 & 138 & 1 & 91 \\
\hline $03 \mathrm{Nov}$ & 21.8 & 4.7 & 8.5 & 13 & 28 & 40 & 35 & 49 & 55 & 296 & 92 & 137 \\
\hline 04 Nov & 29.3 & 7.8 & 4.4 & 16 & 21 & 34 & 26 & 28 & 34 & 379 & 70 & 28 \\
\hline $05 \mathrm{Nov}$ & 20.6 & 0.3 & 2.8 & 12 & 91 & 87 & 23 & 119 & 99 & 146 & 3 & 46 \\
\hline $06 \mathrm{Nov}$ & 19.2 & 8.8 & 4.0 & 13 & 58 & 38 & 26 & 74 & 77 & 147 & 236 & 153 \\
\hline $07 \mathrm{Nov}$ & 33.2 & 3.2 & 1.8 & 17 & 17 & 44 & 33 & 28 & 105 & 366 & 49 & 52 \\
\hline $08 \mathrm{Nov}$ & 27.4 & 2.8 & 4.4 & 13 & 20 & 37 & 25 & 24 & 99 & 358 & 15 & 124 \\
\hline 09 Nov & 28.0 & 2.6 & 5.6 & 13 & 28 & 35 & 29 & 79 & 69 & 298 & 13 & 136 \\
\hline $10 \mathrm{Nov}$ & 36.2 & 3.1 & 3.1 & 14 & 14 & 20 & 24 & 69 & 31 & 350 & 12 & 30 \\
\hline $11 \mathrm{Nov}$ & 11.3 & 0.7 & 1.2 & 12 & 30 & 15 & 22 & 119 & 46 & 113 & 2 & 12 \\
\hline
\end{tabular}

For each start day, five-day simulations were performed with emissions from each of the three source regions reduced in turn on the first day of the run only, as shown in S1. Pulses were derived by subtracting hourly $\mathrm{PM}_{2.5}$ concentrations of each of the 60 five-day runs from the baseline run. Peak and integrated contributions were calculated based on the maxima of the pulse and the area under the curve respectively. A $24 \mathrm{~h}$ running average was applied to the hourly absolute contributions to calculate the timing of the peak contribution. 
Table S2. Scaling factors applied to the 12 emission sources for each of the 60 runs used to generate the emulators.

\begin{tabular}{|c|c|c|c|c|c|c|c|c|c|c|c|c|}
\hline Run No. & Beijing-Industry & Beijing-Power & Beijing-Transport & Beijing-Residential & NN-Industry & NN-Power & NN-Transport & NN-Residential & FN-Industry & FN-Power & FN-Transport & FN-Residential \\
\hline 1 & 0.730 & 1.055 & 0.620 & 0.509 & 0.907 & 0.280 & 0.974 & 0.150 & 0.505 & 0.992 & 0.128 & 0.602 \\
\hline 2 & 0.949 & 0.923 & 1.020 & 0.255 & 0.420 & 0.040 & 1.149 & 1.028 & 0.574 & 0.160 & 1.061 & 0.055 \\
\hline 3 & 0.101 & 0.322 & 0.669 & 0.225 & 0.161 & 1.051 & 0.684 & 0.047 & 0.095 & 0.204 & 0.434 & 0.686 \\
\hline 4 & 0.594 & 0.164 & 0.206 & 0.061 & 1.062 & 0.199 & 0.829 & 0.077 & 0.365 & 0.690 & 0.512 & 0.771 \\
\hline 5 & 0.042 & 0.945 & 1.185 & 0.993 & 0.414 & 0.141 & 0.887 & 0.400 & 1.094 & 1.177 & 0.539 & 1.084 \\
\hline 6 & 0.247 & 1.013 & 0.176 & 1.139 & 1.094 & 1.175 & 0.451 & 0.990 & 0.943 & 0.387 & 0.191 & 0.810 \\
\hline 7 & 0.322 & 0.416 & 0.688 & 0.498 & 1.135 & 0.309 & 0.983 & 0.352 & 0.875 & 0.959 & 0.173 & 1.189 \\
\hline 8 & 0.009 & 0.789 & 0.709 & 0.527 & 0.920 & 0.229 & 0.049 & 0.637 & 0.841 & 1.139 & 0.768 & 1.136 \\
\hline 9 & 0.658 & 0.457 & 0.798 & 0.378 & 0.460 & 0.734 & 0.790 & 0.948 & 1.159 & 0.522 & 0.660 & 1.160 \\
\hline 10 & 0.274 & 0.536 & 0.094 & 0.029 & 0.094 & 0.572 & 0.847 & 0.681 & 0.748 & 0.711 & 0.994 & 0.369 \\
\hline 11 & 0.536 & 0.668 & 0.727 & 0.900 & 0.034 & 0.758 & 1.185 & 1.058 & 0.661 & 0.021 & 0.267 & 0.542 \\
\hline 12 & 0.845 & 1.137 & 0.255 & 0.479 & 0.265 & 0.540 & 0.352 & 0.473 & 0.980 & 0.403 & 0.970 & 0.333 \\
\hline 13 & 0.411 & 0.372 & 1.076 & 0.676 & 0.869 & 0.670 & 0.674 & 0.857 & 0.265 & 1.069 & 0.340 & 0.745 \\
\hline 14 & 0.786 & 0.593 & 0.144 & 0.558 & 1.104 & 0.386 & 0.136 & 0.715 & 1.182 & 0.816 & 0.086 & 1.180 \\
\hline 15 & 0.511 & 0.516 & 0.517 & 0.682 & 0.392 & 0.866 & 0.616 & 0.793 & 0.017 & 0.622 & 0.898 & 0.073 \\
\hline 16 & 1.043 & 0.061 & 0.559 & 1.004 & 0.970 & 0.209 & 0.491 & 0.617 & 0.547 & 0.483 & 0.077 & 0.507 \\
\hline 17 & 0.027 & 0.118 & 0.871 & 0.581 & 0.055 & 1.025 & 0.750 & 1.151 & 0.438 & 0.932 & 0.231 & 0.171 \\
\hline 18 & 0.442 & 0.850 & 0.185 & 0.601 & 0.281 & 0.544 & 1.100 & 0.219 & 1.020 & 0.276 & 1.195 & 0.262 \\
\hline 19 & 0.567 & 0.764 & 0.607 & 0.818 & 0.073 & 0.422 & 0.027 & 0.442 & 0.308 & 0.659 & 1.111 & 0.673 \\
\hline 20 & 1.193 & 0.882 & 0.314 & 0.433 & 1.162 & 0.376 & 0.420 & 0.025 & 0.905 & 0.433 & 1.059 & 1.049 \\
\hline 21 & 0.695 & 0.997 & 1.165 & 0.771 & 0.138 & 1.018 & 1.047 & 0.739 & 0.713 & 0.789 & 1.096 & 0.450 \\
\hline 22 & 1.032 & 0.714 & 0.108 & 0.154 & 0.204 & 1.193 & 0.236 & 0.162 & 1.120 & 0.352 & 0.709 & 0.910 \\
\hline 23 & 0.097 & 0.193 & 0.849 & 0.972 & 0.530 & 0.408 & 0.012 & 0.820 & 0.400 & 1.198 & 1.022 & 0.526 \\
\hline 24 & 0.176 & 0.907 & 0.943 & 0.107 & 0.672 & 0.653 & 0.410 & 0.262 & 0.188 & 0.745 & 0.026 & 0.213 \\
\hline 25 & 0.619 & 1.101 & 0.373 & 0.338 & 0.319 & 0.681 & 0.264 & 0.898 & 0.036 & 0.587 & 0.149 & 0.186 \\
\hline 26 & 0.500 & 0.825 & 0.432 & 0.736 & 0.324 & 0.764 & 0.517 & 1.139 & 0.823 & 0.905 & 1.017 & 0.561 \\
\hline 27 & 0.977 & 0.488 & 0.776 & 1.093 & 1.017 & 0.883 & 1.134 & 0.974 & 0.412 & 0.139 & 0.638 & 0.233 \\
\hline 28 & 0.806 & 0.300 & 0.990 & 1.080 & 1.052 & 0.906 & 1.018 & 0.577 & 1.122 & 0.224 & 0.616 & 0.825 \\
\hline 29 & 1.174 & 0.093 & 0.835 & 0.178 & 0.799 & 0.090 & 0.090 & 0.300 & 0.072 & 0.002 & 0.295 & 0.582 \\
\hline 30 & 0.310 & 1.161 & 0.584 & 0.705 & 1.035 & 0.446 & 0.730 & 1.095 & 0.352 & 0.826 & 0.939 & 0.418 \\
\hline 31 & 0.399 & 1.195 & 1.151 & 0.564 & 0.888 & 0.472 & 0.205 & 1.186 & 0.465 & 0.193 & 0.445 & 0.248 \\
\hline 32 & 0.998 & 0.462 & 0.534 & 0.850 & 0.563 & 0.964 & 0.873 & 1.102 & 0.137 & 0.861 & 0.251 & 0.659 \\
\hline 33 & 0.422 & 0.750 & 0.815 & 1.169 & 0.621 & 0.923 & 1.175 & 0.091 & 0.584 & 0.459 & 0.866 & 1.010 \\
\hline 34 & 0.376 & 0.730 & 0.914 & 0.647 & 0.518 & 1.141 & 0.717 & 0.380 & 0.680 & 0.380 & 0.205 & 0.111 \\
\hline 35 & 1.004 & 0.387 & 0.754 & 0.888 & 0.836 & 1.093 & 0.158 & 0.656 & 0.460 & 0.555 & 1.138 & 0.629 \\
\hline 36 & 0.868 & 0.046 & 0.461 & 1.052 & 0.843 & 0.835 & 0.108 & 0.194 & 0.888 & 0.083 & 0.686 & 0.032 \\
\hline 37 & 1.103 & 0.602 & 0.064 & 1.021 & 0.643 & 0.595 & 0.560 & 0.400 & 0.162 & 0.109 & 0.757 & 0.982 \\
\hline 38 & 0.135 & 0.012 & 0.966 & 0.310 & 0.990 & 0.811 & 1.062 & 0.921 & 0.339 & 0.257 & 0.003 & 0.010 \\
\hline 39 & 1.070 & 0.639 & 0.269 & 1.107 & 0.682 & 1.061 & 0.761 & 0.498 & 0.769 & 1.112 & 0.473 & 0.347 \\
\hline 40 & 0.839 & 0.243 & 0.481 & 0.865 & 0.224 & 0.984 & 1.106 & 0.915 & 0.732 & 0.053 & 0.948 & 0.462 \\
\hline 41 & 1.149 & 0.153 & 0.231 & 0.285 & 0.114 & 1.132 & 0.536 & 0.813 & 0.118 & 1.036 & 0.919 & 1.036 \\
\hline 42 & 0.471 & 0.878 & 0.574 & 1.183 & 0.734 & 0.629 & 0.364 & 0.424 & 0.793 & 0.890 & 1.175 & 0.392 \\
\hline 43 & 0.181 & 0.660 & 1.125 & 0.082 & 0.243 & 0.007 & 0.912 & 0.013 & 0.220 & 0.564 & 0.860 & 1.115 \\
\hline 44 & 0.287 & 1.159 & 0.649 & 0.260 & 1.155 & 0.792 & 0.311 & 0.244 & 0.298 & 0.294 & 0.586 & 0.785 \\
\hline 45 & 0.710 & 0.134 & 0.413 & 0.942 & 0.149 & 0.077 & 0.937 & 0.559 & 0.818 & 0.853 & 0.802 & 0.140 \\
\hline 46 & 0.741 & 0.542 & 1.091 & 0.448 & 0.610 & 0.340 & 0.199 & 0.331 & 0.057 & 0.612 & 1.144 & 0.292 \\
\hline 47 & 0.671 & 0.340 & 1.106 & 0.793 & 0.808 & 1.103 & 0.079 & 0.315 & 0.156 & 1.047 & 0.568 & 0.895 \\
\hline 48 & 0.776 & 1.072 & 0.932 & 0.635 & 0.186 & 0.117 & 0.654 & 0.865 & 0.980 & 1.006 & 0.786 & 0.428 \\
\hline 49 & 0.552 & 0.204 & 0.007 & 0.921 & 0.717 & 0.266 & 0.477 & 1.066 & 1.179 & 0.317 & 0.542 & 0.974 \\
\hline 50 & 0.905 & 0.817 & 0.322 & 0.833 & 0.376 & 0.351 & 0.396 & 0.511 & 0.625 & 0.331 & 0.051 & 1.061 \\
\hline 51 & 0.077 & 0.425 & 0.046 & 0.211 & 0.585 & 0.958 & 0.168 & 0.763 & 0.922 & 0.737 & 0.491 & 0.848 \\
\hline 52 & 0.893 & 0.233 & 0.022 & 0.418 & 0.751 & 0.509 & 0.952 & 1.017 & 0.610 & 0.469 & 0.312 & 0.952 \\
\hline 53 & 0.156 & 0.034 & 1.060 & 0.181 & 0.341 & 0.480 & 0.816 & 0.596 & 1.060 & 0.960 & 0.827 & 0.726 \\
\hline 54 & 1.086 & 0.564 & 0.400 & 0.047 & 0.007 & 0.242 & 0.291 & 0.536 & 0.485 & 1.090 & 0.113 & 0.152 \\
\hline 55 & 0.239 & 0.264 & 0.356 & 0.747 & 1.190 & 0.851 & 0.324 & 0.676 & 0.534 & 0.080 & 0.647 & 0.300 \\
\hline 56 & 0.213 & 1.040 & 0.889 & 0.398 & 0.480 & 0.704 & 0.584 & 0.116 & 1.034 & 0.771 & 0.405 & 0.497 \\
\hline 57 & 0.342 & 0.973 & 0.458 & 0.136 & 0.774 & 0.169 & 0.626 & 1.169 & 0.210 & 0.661 & 0.329 & 0.936 \\
\hline 58 & 0.637 & 0.315 & 1.017 & 0.358 & 0.459 & 0.135 & 0.241 & 0.757 & 0.645 & 1.159 & 0.389 & 0.719 \\
\hline 59 & 0.934 & 0.698 & 0.137 & 1.151 & 0.543 & 0.027 & 0.540 & 0.133 & 0.252 & 0.152 & 0.726 & 0.097 \\
\hline 60 & 1.123 & 1.082 & 0.280 & 0.004 & 0.945 & 0.601 & 1.031 & 0.236 & 1.042 & 0.510 & 0.380 & 0.865 \\
\hline
\end{tabular}

The near-neighbourhood region is labelled NN and the far-neighbourhood region is labelled FN. 
Table S3. Total emission fluxes of key pollutants for each sector within the Far-Neighbourhood region for October 2014.

\begin{tabular}{lccccc}
\hline & Industry & Power & Transport & Residential & Agriculture \\
\hline $\mathrm{CO}\left(\mathrm{mol} \mathrm{h}^{-1}\right)$ & $1.16 \mathrm{e}+06$ & $0.07 \mathrm{e}+06$ & $0.23 \mathrm{e}+06$ & $1.36 \mathrm{e}+06$ & - \\
$\mathrm{NO}\left(\mathrm{molh}^{-1}\right)$ & $8.14 \mathrm{e}+04$ & $9.84 \mathrm{e}+04$ & $5.22 \mathrm{e}+04$ & $1.47 \mathrm{e}+04$ & - \\
$\mathrm{NMVOC}\left(\mathrm{molh}^{-1}\right)$ & $6.92 \mathrm{e}+04$ & $4.33 \mathrm{e}+02$ & $1.04 \mathrm{e}+04$ & $4.40 \mathrm{e}+04$ & - \\
$\mathrm{SO}_{2}\left(\mathrm{molh}^{-1}\right)$ & $9.20 \mathrm{e}+04$ & $4.80 \mathrm{e}+04$ & $1.24 \mathrm{e}+03$ & $4.33 \mathrm{e}+04$ & - \\
$\mathrm{NH}_{3}\left(\mathrm{molh}^{-1}\right)$ & $7.16 \mathrm{e}+03$ & 0.03 & $6.84 \mathrm{e}+02$ & - & $1.47 \mathrm{e}+05$ \\
$\mathrm{PM}_{2.5}\left(\mathrm{~kg} \mathrm{~h}^{-1}\right)$ & $2.74 \mathrm{e}+03$ & $4.48 \mathrm{e}+02$ & $2.07 \mathrm{e}+02$ & $2.83 \mathrm{e}+03$ & - \\
\hline
\end{tabular}

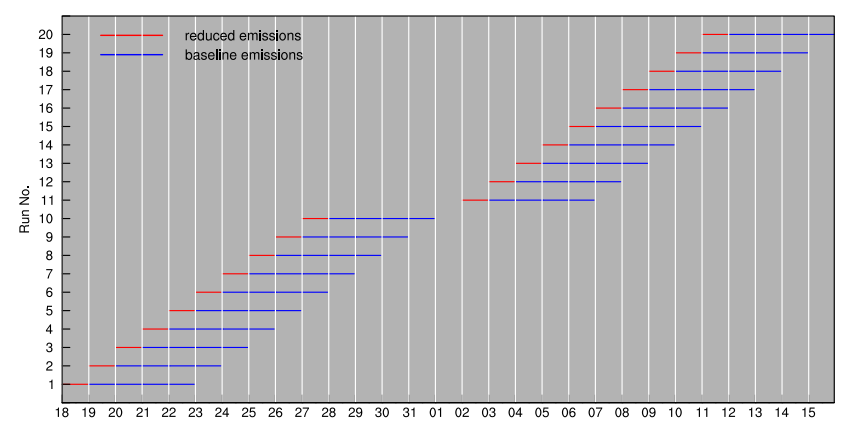

Figure S1. Schematic showing start and end dates of each one-day emission reduction simulation between 12 October and 15 November. These runs were performed for each of the three source regions in turn.
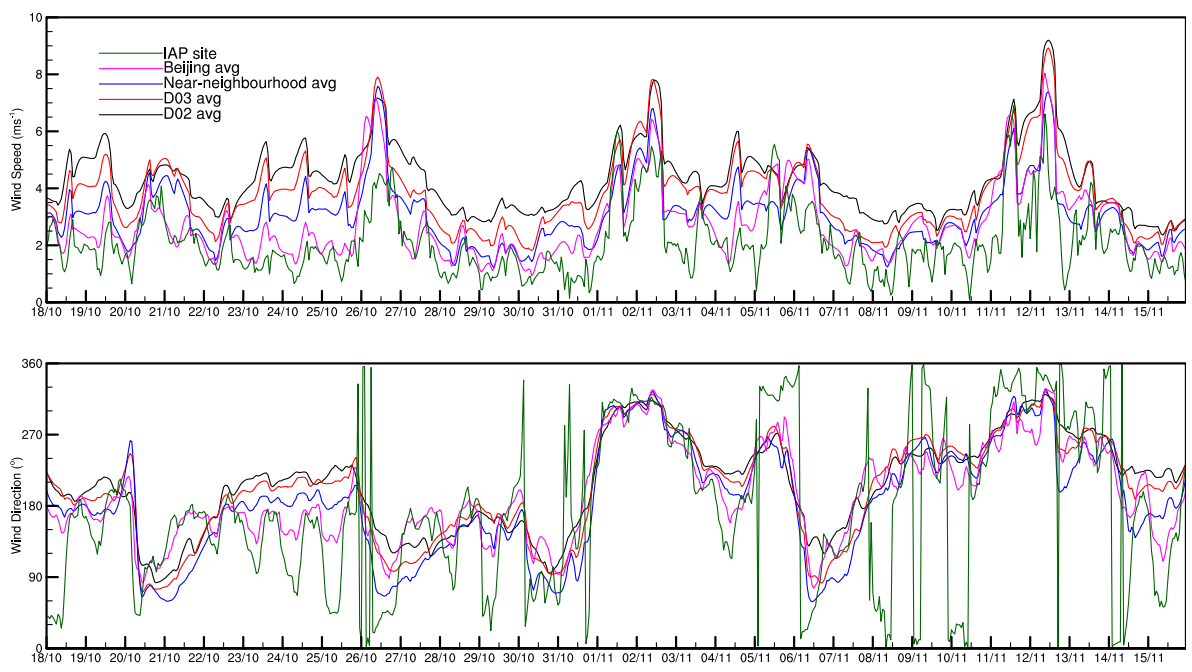

Figure S2. Time series of simulated hourly wind speed and direction sampled from the Institute of Atmospheric Physics (IAP) grid point, and spatially averaged values over Beijing, Near-Neighbourhood region, model domain 2 and 3. Model domain 2 (D02) is the same as shown in Figure 2 and domain 3 (D03) is the same as shown in Figure 8. 


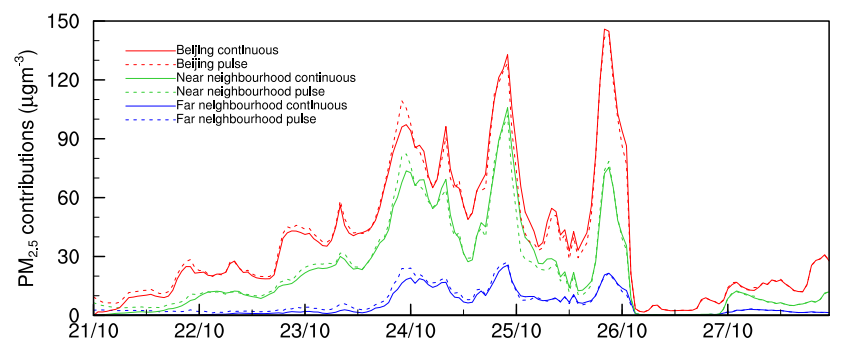

Figure S3. Comparison of cumulative contributions to $\mathrm{PM}_{2.5}$ in Beijing from continuous emission controls (solid lines) with those from successive one-day pulses in emissions (dashed lines) from local, near-neighbourhood and far-neighbourhood source regions.

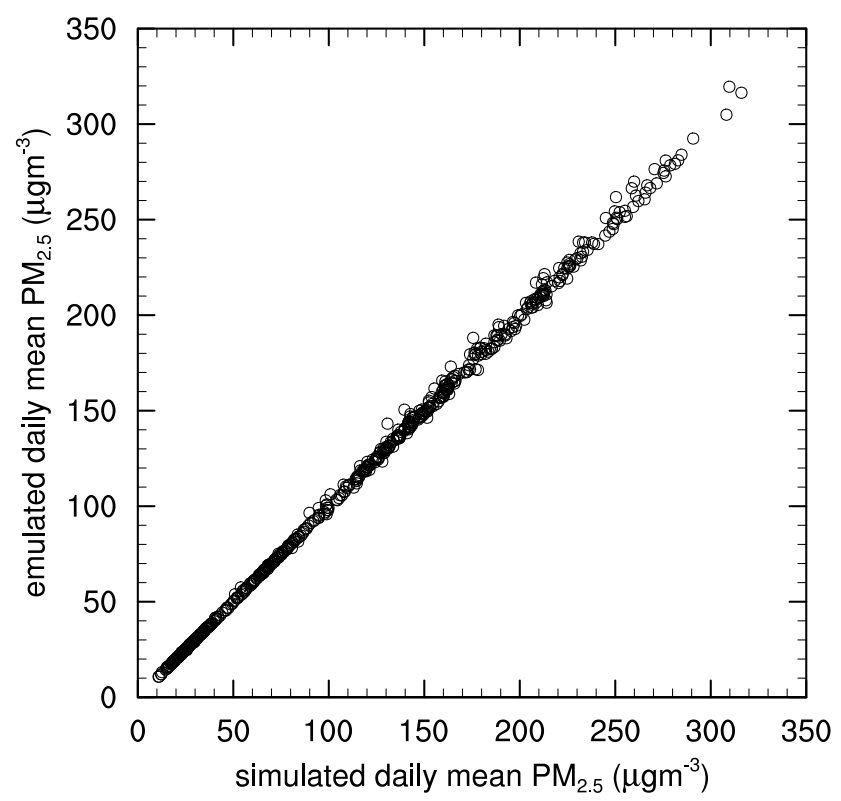

Figure S4. Comparison of simulated versus emulated daily mean $\mathrm{PM}_{2.5}$ in Beijing for emulator validation. For each day of the period 60 different emulators were built leaving out one set of model output and validating against this set; correlation co-efficient $\mathrm{r}=0.999$. 


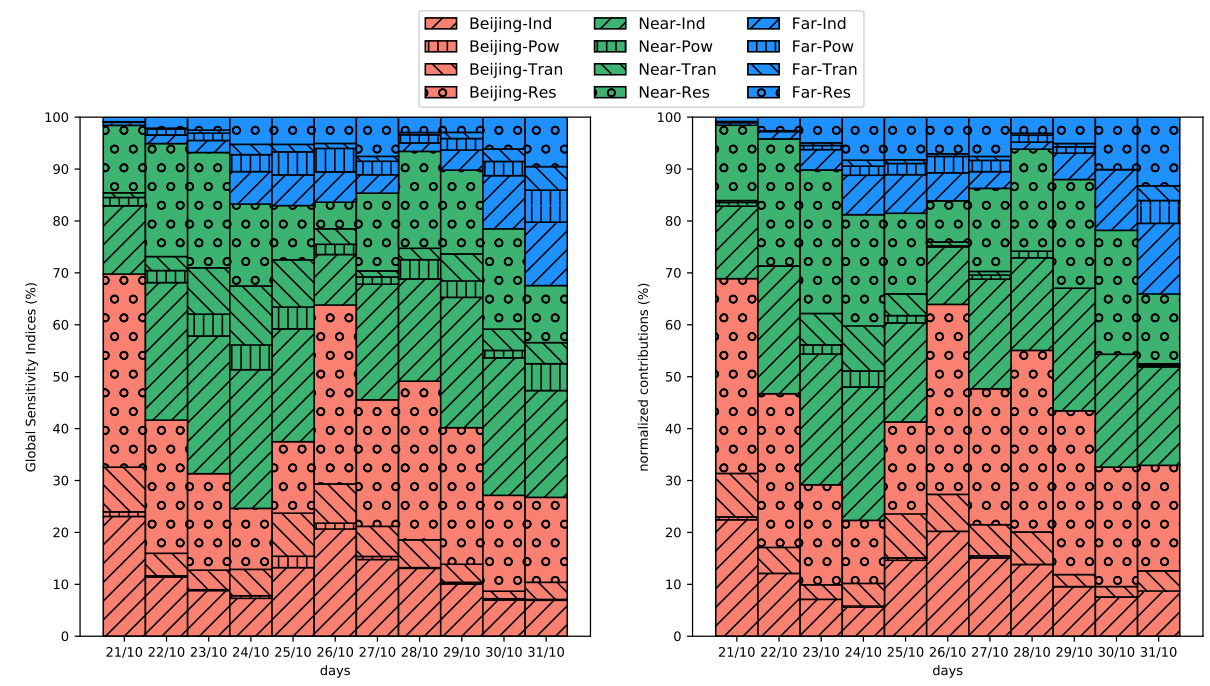

Figure S5. Comparison of contributions to daily mean $\mathrm{PM}_{2.5}$ concentrations from the 12 emission sources derived from global sensitivity analysis of emulated output using the eFAST algorithm (left) and normalized contributions obtained from one-at-a-time sensitivity runs with WRF-Chem (right). 

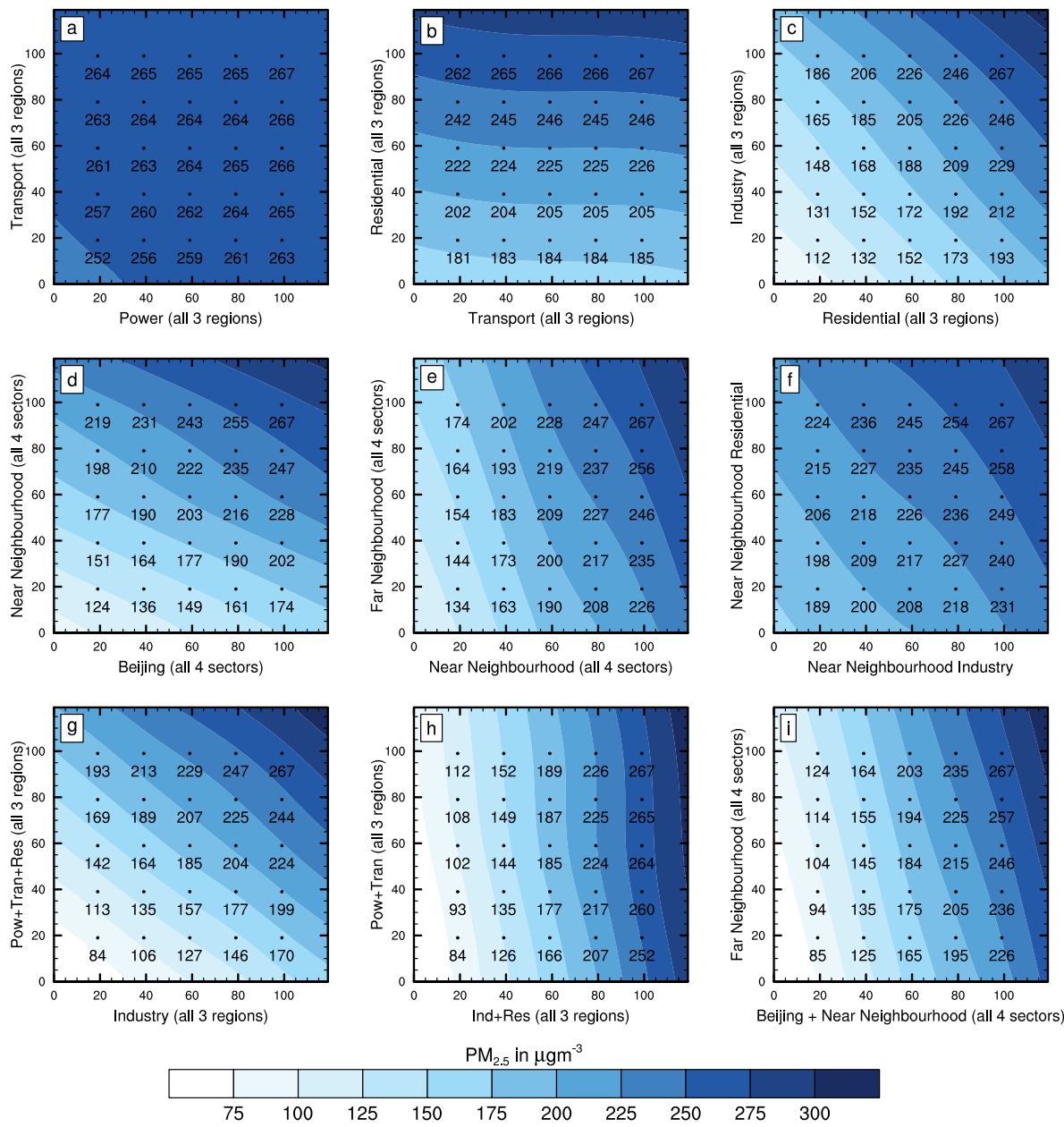

Figure S6. Response of daily average $\mathrm{PM}_{2.5}$ concentrations in Beijing to changes in sectoral and regional emissions. Axes show the scaling applied to the relevant source (in \%) starting on 21 October; contours show the corresponding daily mean $\mathrm{PM}_{2.5}$ in Beijing on 30 October (concentrations at $20 \%$ intervals are labelled). 

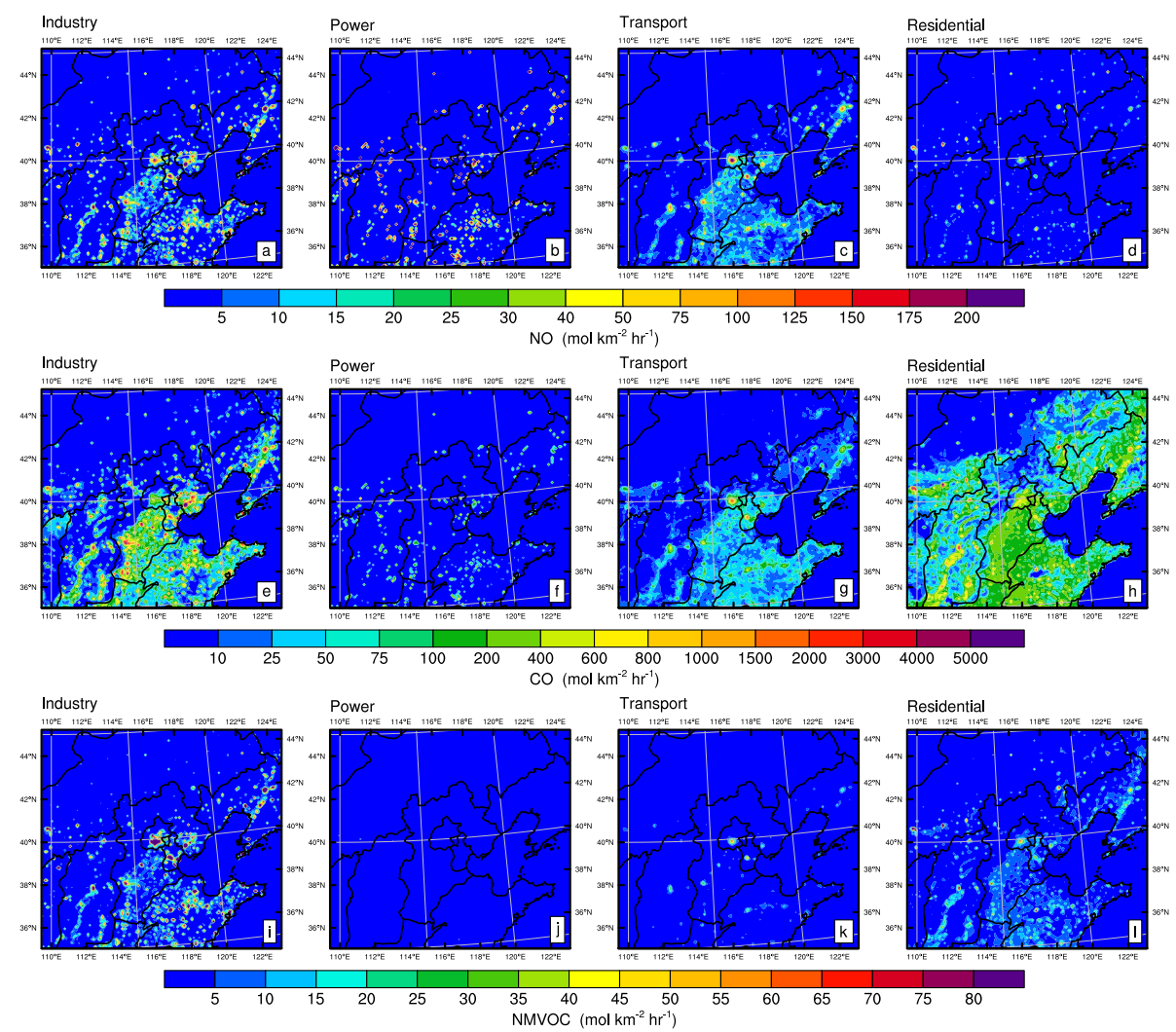

Figure S7. Sectoral emissions of nitrogen dioxide (a-d), carbon monoxide (e-h) and non-methane volatile organic compounds (i-l) for October 2014. 

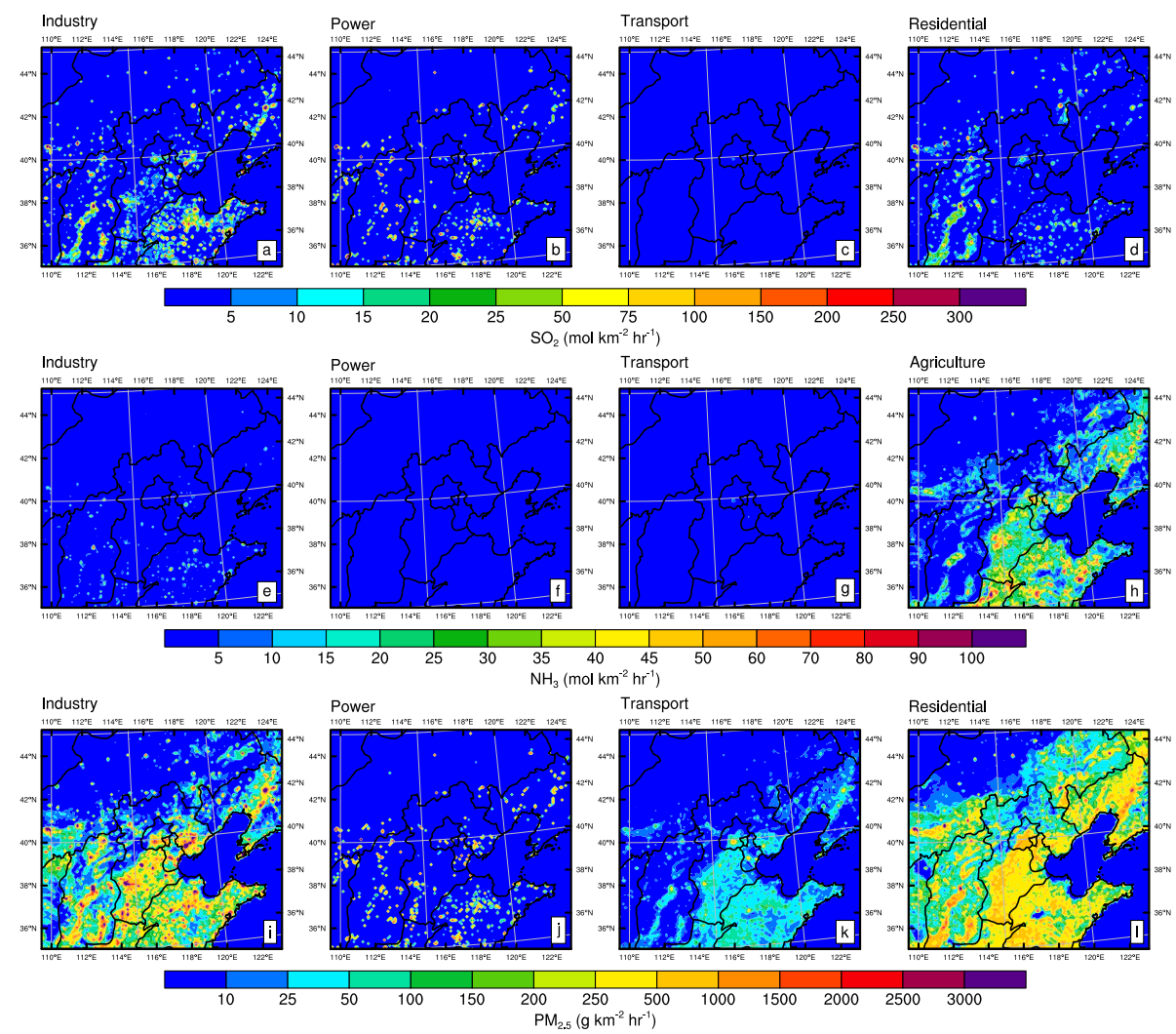

Figure S8. Sectoral emissions of sulfur dioxide (a-d), ammonia (e-h) and $\mathrm{PM}_{2.5}$ (i-l) for October 2014. 